\title{
Spectrum of Histopathological Study of Lesions of Nasal cavity and Paranasal Sinuses - A two Years Study
}

\section{Rajitha $\mathbf{J}^{1^{*}}$, Srikanth $\mathrm{S}^{2}$}

${ }^{1}$ Associate Professor, Department of Pathology, Chalmeda Anand Rao Institute of Medical Sciences, Karimnagar, Telangana, India ${ }^{2}$ Professor, Department of Pathology, Malla Reddy Institute of Medical Sciences, Hyderabad, Telangana, India

*Corresponding author: Dr. S. Srikanth

Abstract

Introduction: The nasal cavity, paranasal sinuses, and nasopharynx though in continuity form a complex system of upper respiratory tract; this region is endowed with a variety of elements such as epithelial, glandular, lymphoid, cartilage, and bone and is also exposed to a variety of infections, tumor-like and true neoplastic conditions. Materials and Methods: The present study is conducted to know the various inflammatory and neoplastic lesions of nasal cavity. The present study is a retrospective and prospective study done done for a period of two years. All the required clinical details were considered in our study. Results: The patients' age ranged from 18 months to 59 years. Majority of the lesions were inflammatory compared to neoplastic and males were commonly affected than females. Conclusion: Amongst the non-inflammatory lesion, nasal polyp is the commonest lesion. The common age group is second and third decades, with male predominance. Among the neoplastic lesions capillary haemangioma was commonest lesion followed by inverted papilloma.

Keywords: Nasal cavity, paranasal sinuses, polyp, hemangioma.

Copyright ( 12021 The Author(s): This is an open-access article distributed under the terms of the Creative Commons Attribution 4.0 International License (CC BY-NC 4.0) which permits unrestricted use, distribution, and reproduction in any medium for non-commercial use provided the original author and source are credited.

\section{INTRODUCTION}

Sinonasal tract is a collective term used for nasal cavity and paranasal sinuses [1]. The main functions of the sinonasal tract are filtering and humidifying inhaled air. The nasal cavity also has specific olfactory receptors for airborne odorant molecules. The paranasal sinuses additionally have the functions of acting as resonating chambers during speech and reducing the relative weight of the skull. These functions lead to exposure of various allergens, pathogens, chemical and physical irritants and other environmental influences. As a result of these multifaceted exposures, various inflammatory conditions, infections and Neoplasms can occur in the sinonasal tract [2].

A variety of non-neoplastic and neoplastic conditions involve the nasal cavity (NC), paranasal sinuses (PNS) and these are very common lesions encountered in clinical practice. A large number of diseases affecting these structures are due, to many of the specialized tissues, each with its own aberrations that exist in the region. The presenting features and symptomatology and advanced imaging technique help to reach a presumptive diagnosis but histopathological examination remains the mainstay of definitive diagnosis. Thus careful histological workup is essential for a correct diagnosis and timely intervention. The aim of this clinicopathological study was to find out the incidence of non-neoplastic and neoplastic lesions of NC and PNS, mode of presentation and histological types and to find out the great variety of non-neoplastic and neoplastic lesions of NC and PNS.

\section{Materials ANd MeTHOdS}

The present study is a retropective and prospective study done for a period of two years. All clinical history, radiological and clinical details were considered. All biopsies were submitted to department of Pathology. Sections were submitted for routine grossing and staining done and examined under microscoipe.

\section{RESULTS}

A total of 56 cases were included in the present study. Out of the 56 cases Allergic inflammatory polyp constituted highest number of cases. Other lesions like chromic nonspecific inflammation, papilloma, hemangioma, fibroma, 
olfactory neuroblastoma and angiofibroma were there [Table 1]. Male patients constituted highest number of cases and age group between 31-40 years constituted highest number of cases [Table 2].

\section{DISCUSSION}

The nose is the most prominent part of the face with substantial aesthetic and functional significance. It is one of the few organs of body invested with an aura of emotional and cultural importance. Anatomical location of the nose and it passage have been regarded as the direct avenue to the brain, man's source of intelligence and spirituality.

Prevalence of sinonasal masses in the general population is 1 to $4 \%$ [3]. A careful clinical workup including symptomatology, radiological investigations and endoscopy helps to determine a differential diagnosis but histopathology provides the final diagnosis [4]. Thus histopathological examination is essential for timely diagnosis and intervention [5].

The most common symptom at presentation was unilateral nasal obstruction while an initial clinical diagnosis of chronic sinusitis was made in nearly one third of cases. Unilateral or bilateral nasal obstruction, blood-tinged nasal discharge, anosmia, diplopia, facial numbness, and facial swellings were the clinical features that favored a malignant sinonasal tumor. The maxillary sinus was the most common site of involvement in all lesions. CT and MRI scans were utilized to delineate tumor extent and bone destruction. Management was based on the anatomical site of origin and its extent, and histopathologic diagnosis. Even benign tumors had a tendency for local recurrence and bone destruction.

Nasal polyps are the most common benign tumors of the nasal cavity, as was seen in our study. They result from chronic inflammation, allergens, pollutants, infectious agents, chronic inflammation, and cystic fibrosis. There is no particular age or sex predilection $[6,7]$. In our study, males were slightly more affected. For imaging, coronal sinus CT and endoscopy were used and for treatment NSAIDs, corticosteroids, and endoscopy-guided polypectomy.

Nasal polyps result from chronic inflammation of the nasal and sinus mucous membranes and are the most common tumours of the nasal cavity. Their exact pathogenesis is not known, however a strong association with allergy, infection, asthma and aspirin sensitivity has been implicated. We found $71.4 \%$ of the sinonasal masses to be non-neoplastic. Such a high proportion of non-neoplastic lesions have been reported in previous studies. Nasal polyp was the most common non-neoplastic mass and was similarly documented by those authors. True nasal polyps are subdivided into allergic nasal polyps, showing abundant eosinophils in the stroma in addition to inflammatory cells, whereas in the other type viz. inflammatory nasal polyps, there is a paucity of eosinophils. Ethmoidal and antrochoanal polyps are generally allergic and inflammatory in nature, respectively.

Peak incidence of sinonasal polyps was noted in the third decade. Similar findings were observed by Khan et al. and Kulkarni et al. who reported a peak incidence in second and third decade of life. Microscopically, the epithelial lining is ciliated pseudostratified columnar, with stromal infiltrate comprising of eosinophils, lymphocytes and neutrophils.

Papillomas and inverted papillomas tend to be multicentric in up to $30 \%$ of cases and $5-20 \%$ of cases undergo malignant transformation. Hemangiomas are benign neoplasms of vascular origin with a marked male preponderance (approximately 6:1). Most cases in our study were capillary hemangiomas with almost no sex predilection.

Histopathological examination is conclusive in diagnosing the polypoid lesions, describing both aetiology and cellular details. It is the only means of determining the nature of the disease, i.e. inflammatory or neoplastic. Radiological investigations may also help in understanding the type of pathology, extension of lesion and associated sinus pathology. Most of nonneoplastic and benign neoplastic nasal masses require surgical excision, while malignant neoplastic nasal masses require wide surgical excision, radiotherapy or chemotherapy either alone or in combination. Regular follow-up is necessary for early detection of recurrence or metastases. The outcome for malignant lesions is relatively poor and associated with late diagnosis, difficult surgical anatomy and a lack of effective adjuvant modalities of treatment. Topically applied corticosteroids have a favourable effect on symptoms. However, corticosteroids are only helpful in allergic conditions. FESS was the most commonly used surgical intervention (44.6\%) followed by excision of the mass (25.0\%). Polypectomy and Caldwell-Luc procedure for nasal polyposis are certainly associated with a risk of recurrence. FESS offers a definite advantage over other procedures and is now the preferred modality. Complete surgical resection followed by adjuvant radiotherapy is an effective and safe approach in the treatment of sinonasal cancer and associated with better survival. However, radiotherapy was the only mode of treatment offered to patients in our clinic.

Olfactory neuroblastoma is a rare malignant tumor believed to originate in olfactory cells most commonly presented as unilateral polypoid nasal masses often causing nasal obstruction and epistaxis. The olfactory cells, located in the upper rear of the inside of the nose, are responsible for the sense of smell. It has a predilection for invading surrounding regions, such as the paranasal sinuses, orbits skull base 
and can cause loss of smell, taste, and vision, as well as facial disfigurement in advanced cases. It is a rare disease that was first described in 1924 in the French medical literature and since that time, this tumor continues to be uncommon, incidence being less than $5 \%$ of all sinunasal malignancies.

Olfactory neuroblastoma is a neuroendocrine tumour capable of causing paraneoplastic syndrome by secreting peptides. ONB often responds well to radiation therapy, but the tumor has a high tendency to recur after excision. Olfactory neuroblastoma is considered distinct from other neuroblastomas, because it does not originate in the sympathetic nervous system.

This tumor has a broad histological spectrum and olfactory neuroblastoma can be confused with other small round cell tumors and undifferentiated carcinomas.

Some data indicate that various industrial exposures may be related to cancer of the paranasal sinus and nasal cavity. The risk of a second primary head and neck tumor is considerably increased. A subgroup has shown that paranasal sinus and nasal cavity SCCs are associated with human papilloma virus (HPV) infection and that HPV-positive patients may have a better prognosis than those who are HPV negative.

\section{CONCLUSION}

The nasal cavity and paranasal sinuses are exposed to many environmental pollutants and pathogens. Exposure to such influences can lead to a variety of lesions primarily affecting the sinonasal tract. The aim of this study was to find out the incidence, age and sex distribution and to enlist the different types of lesions of the nasal cavity and paranasal sinuses.

Definite diagnosis is essential for further management and to determine the prognosis of the patient. Therefore histopathology plays an intergral role and remains the gold standard for establishing the diagnosis of sinonasal tract lesions.

Table-1: Showing various lesions in the present study

\begin{tabular}{|l|l|l|}
\hline SI No & Lesion & Number of cases \\
\hline 1 & Allergic polyp & 24 \\
\hline 2 & Chronic non-specific inflammation & 10 \\
\hline 3 & Papilloma & 08 \\
\hline 4 & Hemangioma & 08 \\
\hline 5 & Fibroma & 04 \\
\hline 6 & Olfactory neuroblastoma & 01 \\
\hline 7 & Angiofibroma & 01 \\
\hline
\end{tabular}

Table-2: Table showing various age groups in the present study

\begin{tabular}{|l|l|l|}
\hline SI No & Age group & Number of cases \\
\hline 1 & $0-10$ & 01 \\
\hline 2 & $11-20$ & 09 \\
\hline 3 & $21-30$ & 06 \\
\hline 4 & $31-40$ & 33 \\
\hline 5 & $41-50$ & 06 \\
\hline 6 & $51-60$ & 01 \\
\hline
\end{tabular}

\section{REFERENCES}

1. Pilch BZ, Bouquot J, Thompson LD. Squamous cell carcinoma. In: Barnes EL, Eveson JW, Reichart P, Sidransky D, editors. Pathology and genetics of head and neck tumours. (Kleihues P, Sobin LH, series editors). World Health Organization classification of tumours. Lyon, France: IARC Press. 2005: 15-17.

2. Hopkin N, McNicoll W, Dalley VM, Shaw HJ. Cancer of the paranasal sinuses and nasal cavities. Part I. Clinical features. J Laryngol Otol. 1984. Jun;98(6):585-595.

3. Kirtsreesakul V. Update on Nasal polyps: Etiopathogenesis. J Med Assoc Tahi. 2005;88(12): 1966-1972.

4. Zafar U, Khan N, Afroz N, Hasan S. Clinicopathological study of non-neoplastic lesions of nasal cavity and paranasal sinuses. Indian $\mathrm{J}$ Pathol Microbiol. 2008;51(1):26.

5. Kulkarni A, Mudholkar V, Acharya A, Ramteke R. Histopathological Study of Lesions of Nose and Paranasal Sinuses. Indian J Otolaryngol Head Neck Surg. 2012;64(3):275-279.

6. Lund VJ. Diagnosis and treatment of nasal polyps. BMJ. 1995. Nov;311(7017):1411-1414.

7. Lathi A, Syed MM, Kalakoti P, Qutub D, Kishve SP. Clinico-pathological profile of sinonasal masses: a study from a tertiary care hospital of India. Acta Otorhinolaryngol Ital. 2011. Dec;31(6):372-377. 